The indications for, and the results of, various peripheral procedures that give temporary relief are summarized, and drug treatment is mentioned.

There is no single treatment for all cases of trigeminal aeuralgia. Some patients will not tolerate injection and others are afraid of operation. There are individual considerations in svery case, and the best treatment may be operation, or Gasserian injection, or peripheral-nerve injection, or tablets ; all have a place in proper management and all should be considered by the surgeon who has experience of all methods and is familiar with the Gasserian region.

It is a pleasure to thank Dr. D. G. F. Harriman, neuropathologist, tor his helpful comments about pathology, and all former assistants for their case records ; also to thank the patients and their doctors for their willing co-operation.

\section{REPERENCES}

Cushing, H. (1920). Amer. F. med. Sci., 160, 157.

Dott, N. M. (1951). Proc. roy. Soc. Med., 44, 1034

Fothergill, J. (1773). Med. Obs. Inquir., 5, 129.

Frazier, C. H. (1931). F. Amer. med. Ass., 96, 913.

- and Spiller, W. G. (1901). Philad. med. f., 8, 1039.

Harris, W. (1912). Lancet, 1, 218.

(1940). Brain, 63, 209.

Henderson, W. R (1965). Ann, roy. Coll. Surg. Engl., 37, 346.

Henderson, W. R. (1965). Ann. roy. Coll.

King, R. B., Meagher, J. N., and Barnett, J. C. (1950). 7. Neurosurs., 13, 176.

Kugelberg, E., and Lindblom, U. (1959). ₹. Neurol. Neurosurg. Psychiat., 22, 36.

Penman, J. (1953). Lancet, 1, 760.

Rushton, J. G., and Olafson, R. A. (1965). Arch. Neurol. (Chic.), 13, 383.

Schorstein, J. (1943). F. Neurol. Psychiat., 6, 46.

Spillane, J. D. (1964). Practitioner, 192, 71.

Trousseau, A. (1868). Lectures on Clinical Medicine, vol. 1, p. 105 London.

\title{
Results of No-loop Gastrectomy for Duodenal Ulcer
}

\author{
HERMON TAYLOR,* M.D., M.CH., F.R.C.s ; D. G A. EADIE,* F.R.C.S
}

Briz. med. 9., 1967, 1, 15-18

The operation of partial gastrectomy for duodenal ulcer has been in general use in this country for many years. In most cases satisfactory relief has been obtained from the burden of the ulcer, but the quality of this benefit has been modified by the vulnerability of the stoma to recurrent ulceration, by the so-called postgastrectomy syndromes, and by some reduction in the level of general nutrition. The latter is due partly to the inadequacy of gastric digestion during the transient passage of the meal through the remnant of the stomach, but also to the virtual exclusion of the duodenum and upper jejunum from the path of the food that is brought about by the Polya type of gastrectomy.

By practising a rather extensive gastric resection the incidence of stomal ulceration has been controlled, though at the cost of accentuating the difficulties of nutrition. The most slusive problem, however, has been that of the postgastrectomy syndromes. This has not been made easier by the word "dumping," which has been stretched from the conception of overloading of the jejunum distal to the gastric anastomosis to cover the quite different problem of obstruction on the proximal side of the stoma. In mild cases this may involve no more than postprandial nausea and sweating, but in more severe cases the discomfort persists between meals, with distension, loose stools, and attacks of bile-vomiting. The final state can be one of complete misery and malnutrition-even tuberculosis.

The inexplicable and tantalizing question about these obstructive manifestations is why they should occur in one patient and not in the next-patients who, so far as one can tell, have undergone the same surgical procedure. The evidence suggests that they are due to the displacement of the proximal loop of jejunum from under the mesocolon up to the gastric stump in order to make the anastomosis. This attachment creates a variable degree of obstruction, partly by simple kinkIng, partly by rotation effects at the stoma (Stammers, 1954 ; Tanner, 1954 ; Hermon Taylor, 1964). The intensity of the symptoms depends on the degree of obstruction, but if dilatation and stasis allow abnormal bacterial growth to occur malabsorption may follow in the whole of the distal intestine (Stammers, 1961 ; Orr, 1964). The appropriate name for these phenomena is therefore not the postgastrectomy syndrome but the loop-

\footnotetext{
- The London Hospital.
}

gastrectomy syndrome-a phrase which places the responsibility for them on the jejunal loop proximal to the anastomosis rather than on the gastric resection as such.

The reality of this conception was illustrated in one of our patients who was himself a surgeon with experience of gastrectomy. He had already had a partial gastrectomy for duodenal ulcer and two revision operations for "dumping " at which, however, the jejunal loop had been retained. He described two quite different types of pain-one after eating too much, and the other, " a more distressing type radiating into the back and chest, which seemed to have no anatomical basis." At his final operation the jejunal loop was detached from the stomach and returned to its anatomical position and a no-loop anastomosis was made (see below), with subsequent relief from the symptoms. The special interest in this case concerns a naso-oesophageal catheter which had been guided through the stomach and the stoma into the duodenum as a safeguard against postoperative distension. Before this tube was removed some days later it was used to study the function of the parts under $x$-ray screening. It was found that the injection of $20 \mathrm{ml}$. of Gastrografin into the duodenum produced an acute discomfort which the patient recognized as the same as that which had been associated with his jejunal loop. The pain was relieved by withdrawing the fluid from the duodenum. The catheter was then drawn back into the stomach, where a similar injection now produced no sensation, and the medium passed on into the efferent jejunum. This patient is quite convinced that the nauseating discomfort he used to experience with his jejunal loop was due to duodenal distension.

\section{The "No-loop" Operation}

The object of this paper is to show that a design of gastrectomy which does not employ a jejunal loop for the anastomosis (Hermon Taylor, 1959) is not only free from this obstructive syndrome but also, by restoring a measure of duodenal function, affords some protection of the stoma from gastric acid and a better level of nutrition than the standard operation. Fig. 1 shows how the "no-loop" anastomosis functions in this way and indicates the difference from the loop gastrectomy (Fig. 2). By freeing the fundus of the stomach from the spleen the anastomosis can be made to the duodeno- 
jejunal junction at the ligament of Treitz, or just proximal to it (W. M. Capper, personal communication). This allows the jejunum to remain undisturbed in its anatomical situation free from any obstructive attachments. In addition, the proximal position of the stoma diverts the gastric contents into the duodenum, where the acid chyme elicits the normal hormonal responses. These are twofold-the stimulation of the flow of bile and pancreatic juice, and the inhibition of acid secretion in the stomach (Jchnston and Duthie, 1964). Increase of alkaline isecretion and diminution of acid thus combine to protect the stoma from peptic ulceration, and the mixing of food with a normal output of enzymes in the duodenum conserves a measure of physiological digestion there.

\section{Follow-through Material}

The practical results of these considerations are presented in this paper. During the past 20 years 351 "no-loop" gastrectomy operations have been performed for duodenal ulcer

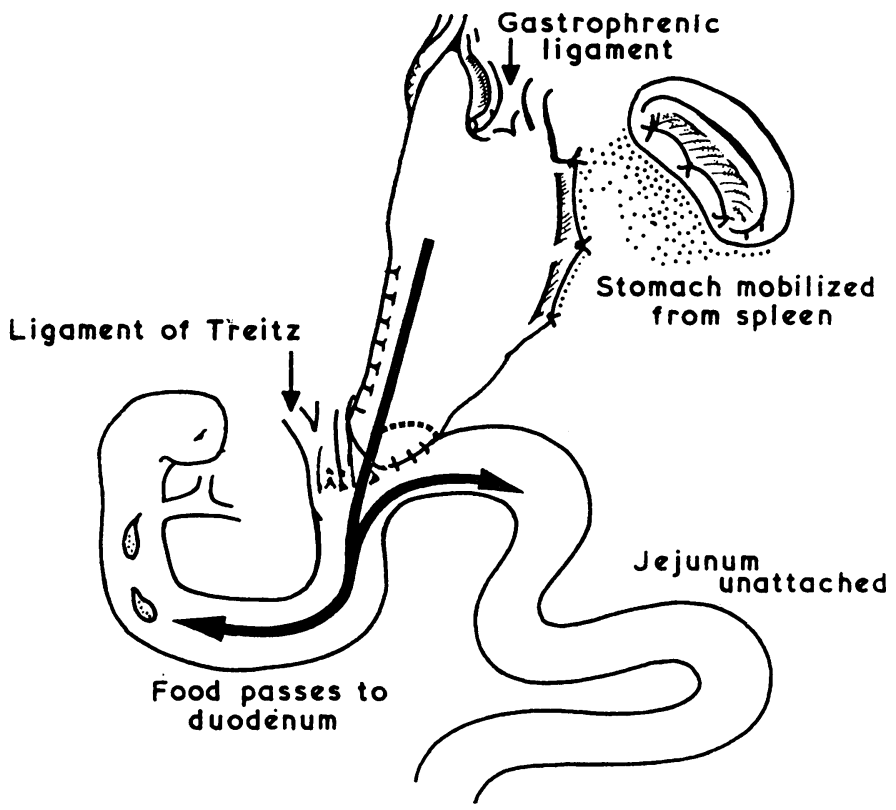

Fig. 1.-No-loop gastrectomy.

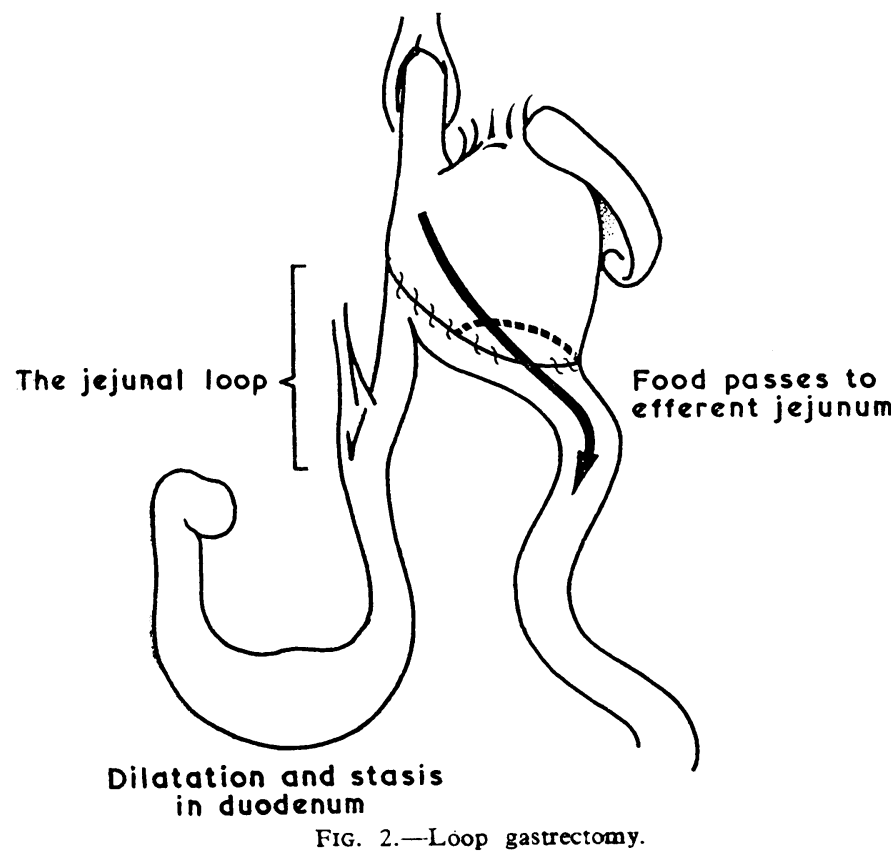

at the London Hospital and King George V Hospital, onethird of which have been carried out by registrars. Of the patients $313(89 \%)$ were men with an average age of 45 years. and $38(9 \%)$ were women, average age 50 .

In $309(88 \%)$ cases the "no-loop" operation was the primary surgical procedure for the duodenal ulcer. The average duration. of symptoms had been 13 years, and many of the patients had experienced one or more complications, such as bleeding, perforation, or stenosis. No deaths followed any of these primary operations.

In $22(6 \%)$ cases the "no-loop" gastrectomy was a revision procedure in patients with a stomal ulcer or an obstructive syndrome following some other primary operation for duodenal ulcer. One patient in this group died 17 months postoperatively after a short period of fever and jaundice. Post-mortem examination showed multiple abscesses in the liver due to rupture into the splenic vein of an old inspissated abscess over the tail of the pancreas, presumably dating from the operation.

In $20(6 \%)$ cases the gastrectomy was done under emergency conditions for bleeding or perforation. One further death occurred in this group in a patient whose collapse from haemorrhage was so profound that intra-arterial transfusion on the table failed to save him. Post-mortem examination showed an islet-cell tumour of the pancreas.

As $13(3.7 \%)$ patients were lost to follow-through, usually as the result of emigration, there are 336 patients available for review.

Eighteen patients died during the follow-through period. In three cases the cause was not discovered, but in the remaining 15 it was known to be unrelated to peptic ulcer or to the operation (Table I). The condition of these 18 patients was, however, recorded up to the time of their death and they have been included in the total of 336 follow-through results.

TABLE I.-Causes of 18 Deaths During Follow-through Period

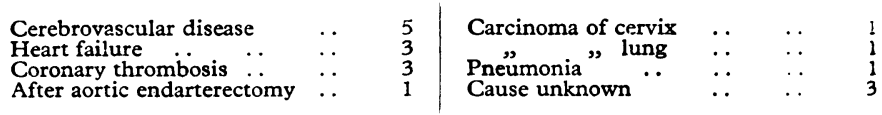

We would like to emphasize the length of the period covered by this review. More than half of the cases have been observed for longer than 10 years and no case has been included in which the operation was performed less than three and a half years ago (Table II).

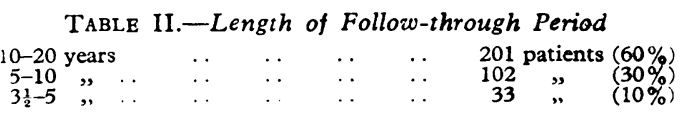

\section{Present Investigation}

Our approach to this investigation has been to look for any patients with symptoms comparable to those of afferent-loop obstruction or anastomotic ulcer which may complicate loop gastrectomy, and also any cases similar to the diarrhoea or recurrent duodenal ulcer which may complicate some cases of vagotomy. Our starting-point was to accept that loss of pyloric function renders the patient liable to symptoms from too rapid entry of a meal into the intestine. We have been interested to record how the patients adapt themselves to this limitation, but our main concern has been to find any incidence of symptoms of obstruction over and above the pattern of meal-time restrictions which this involves.

With regard to colonic function words such as "constipation" or "normal" have been rejected, and the patients have been pressed specifically to say whether or not they pass firm, brown, sausage-shaped stools. Investigations have been confined to those patients whose answer to this question was negative. 
We recognize that it is possible for a solid stool to contain a small excess of fat, but we were concerned throughout only with the practical results of no-loop gastrectomy. The patients vere seen from time to time over the years, but we interviewed most of them within the past 12 months with this report in view. The information is presented under the following headlings.

\section{Discomfort alter Food}

After operation the patients were warned-and soon dissovered for themselves-that they must not eat too much, too quickly ; that they would be wise not to drink at mealtimes; ind that too much of the sweet course might upset them. All but two of the 336 patients were able to avoid discomfort after neals in this way. They varied greatly, however, in their physical and psychological reactions to these limitations. The physical scale lay between those who maintained that they sould eat a three-course meal with a pint of beer and the much nore typical patients who enjoyed their meat and vegetables but avoided the sweet course and had their cup of tea later. Psychologically the extent to which they obeyed the rules varied with their personalities. The careful ones took no chances and ate strictly within their limits. The unintelligent ones had to be reminded of the rules every time they came to the followthrough department. The impatient ones of ten ate more than their capacity because they were hungry, and accepted a degree of distension and lassitude afterwards as more acceptable than the bother of arranging smaller and more frequent meals. Relaxation and reclining after a meal seemed to be an important factor in digestive comfort. Most patients said that on Sunday, when they can rest after a meal, they can indulge their appetites and enjoy themselves without discomfort, whereas on weekJays the conditions of their employment compelled them to limit their intake until their evening meal. Several patients had changed their jobs to get more favourable conditions for neals during the day, but no patients were unable to work for these reasons.

It is impracticable to try to put figures to this varying pattern of life after no-loop gastrectomy. The information above had nostly to be extracted from the patients by cross-examination. Their point of view was that they could do their work and chey could eat meat and vegetables, and they regarded their limitations beyond this as no great hardship. The two patients who always get discomfort, however carefully they eat, are both underweight and could be said to approach the picture presented by loop obstruction. However, both of them are at work, both pass formed stools, radiology shows unrestricted slling of the duodenum and jejunum, and we can only suppose they have a special sensitivity of the intestine to the entry of food. We found no other examples of intractable distress after food comparable to that of afferent-loop obstruction.

There bas been no case of recurrent ulceration or ulcer-type Iyspepsia.

\section{Bile Vomiting}

About 10 years ago, in the middle of the period covered by this report, we became aware that some of our patients recently sperated on were liable to regurgitation of food, sometimes mixed with bile. The regurgitation occurred if the patients exerted themselves soon after eating and especially on bending. It was associated with heartburn, but not with nausea, and on $x$-ray four of these patients were shown to have developed oesophageal reflux. We realized that the mobilization of the stomach, which at that time we had begun to take right up to the oesophagus, was destroying the oesophago-gastric angle and the competence of the cardia against regurgitation. The mobilization was therefore restricted to the separation of the fundus from the spleen but leaving the gastrophrenic ligament intact. This important membrane lies between the oesophagus and the spleen, holding the proximal $1 \frac{1}{2}$ in. $(3.8 \mathrm{~cm}$.) of fundus to the diaphragm, and so maintaining the oesophago-gastric angle (Fig. 1). Since this modification was introduced there have been no further cases of regurgitation. We have had no case of vomiting of bile unrelated to meals and associated with mounting nausea and discomfort which is characteristic of afferent-loop obstruction.

\section{Bowel Action}

Of the 336 patients 313 (93\%) pass one or two formed firm brown stools a day or are constipated, 20 stated that the stools might be loose on occasion, and 3 said they rarely passed a solid motion (Table III). The latter 23 patients were investigated for steatorrhoea in the metabolic ward of the London Hospital. On a standard three-day collection the fat content of the stools of the 20 patients was found to be less than $5 \mathrm{~g}$. a day. The other three showed frank steatorrhoea with values above this level. In two of the latter, however, the diarrhoea had preceded the no-loop gastrectomy. In one case it had arisen after a previous vagotomy and gastroenterostomy, and the gastrectomy was done for anastomotic ulcer. In the other it was associated with pancreatic deficiency since childhood, and was successfully controlled by pancreatin. The third patient with steatorrhoea was unwilling to lose time at his work to undergo further investigation.

TABLB III.-Bowel Action of 336 Patients

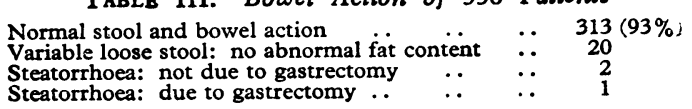

\section{Nutrition}

$\bullet$

Since the operation 18 patients $(5 \%)$ have put on weight, 89 $(26 \%)$ have lost weight, and $229(69 \%)$ have remained about the same. It is difficult to interpret these figures in view of the overweight condition of some patients on milk diets and the wasting of some others with duodenal stenosis before operation. A noticeable fact was the regularity with which patients put on weight after they retire from working life, and it would seem that they were previously underweight simply from lack of proper opportunities to eat in the environment of their employment. There has been no case of gross wasting comparable to that which may result from malabsorption due to obstruction of a jejunal loop.

There were $10(3 \%)$ cases of tuberculosis in the series: five were known to exist before the operation and five came to light afterwards. They all responded to treatment without mortality.

\section{Anaemia}

There was a considerable incidence of anaemia among these patients. Nowadays we advise every gastrectomy patient, and especially the women, for the rest of their lives, to take one tablet of iron every day. However, this advice was not given to the bulk of the patients in this series and they were treated if and when they became anaemic. On this basis 76 patients $(23 \%)$ were clinically thought to be anaemic and their haemoglobin values were estimated : 43 were found to be above $80 \%$ and responded readily to iron therapy, 26 had readings between $60 \%$ and $80 \%$, and 7 were below $60 \%$. The 33 patients with values below $80 \%$ were further investigated (Table IV) ${ }^{1}: 24$ had a simple deficiency of iron, eight had a further deficiency of vitamin $B_{12}$, and one appeared to have a folate deficiency as well. All the patients responded to replacement therapy.

\footnotetext{
${ }^{1}$ We are grateful to Dr. A. L. Turnbull for his investigation and man-
} agement of these cases of anaemia. 
TABLE IV.-Investigation of Anaemia Below $80 \%$ Haemoglobin

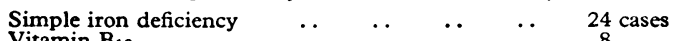
Vitamin $B_{12}$
$\Rightarrow$

\section{Discussion}

The absence of obstructive sequelae in these cases supports the view that when they occur after forms of gastrectomy in which a jejunal loop has been used for the anastomosis the loop is indeed the obstructive agent. It suggests also that the general adoption of the no-loop technique would remove the threat of the so-called postgastrectomy syndrome as a risk incurred by partial gastrectomy in the treatment of duodenal ulcer.

The small incidence of diarrhoea and the absence of recurrent ulceration compare favourably with both vagotomy and loop gastrectomy. Limitation of meals and vulnerability to anaemia can be contained by adequate measures. Nevertheless, they do constitute a real disadvantage. We think that they are both due to the rather extensive (three-quarters) resection of the stomach that has been carried out in this series. This has been done on the supposition that it was necessary to avoid stomal ulceration, but the results suggest that this may not be so. It is probable that the no-loop technique will permit the retentios of a greater proportion of the stomach with less disturbance of function afterwards.

\section{Summary}

The no-loop technique in partial gastrectomy for duodenal ulcer avoids the liability to postgastrectomy syndromes, reduces the risk of recurrent ulcer, and preserves nutrition by maintaining a measure of physiological digestion in the duodenum.

\section{REFERENCES}

Johnston, D., and Duthie, H. L. (1964). Gut, 5, 573.

Orr, I. (1964). Ann. roy. Coll. Surg. Engl., 34, 314.

Stammers, F. A. R. (1954). Brit. F. Surg., 42, 34.

- (1961). Ibid., 49, 28.

Tanner, N. C. (1954). Postgrad. med. F., 30, 448

Taylor, H. (1959). Brit. med. F., 1, 1133.

(1964). Proceedings of International Congress of Gastroenterology. vol. I, p. 218.

\title{
Renal Artery Stenosis with Hypertension and High Haematocrit
}

\author{
PETER HUDGSON,* M.B., M.R.A.C.P. ; JOHN M. S. PEARCE,*† M.D., M.R.C.P. \\ W. KEITH YEATES, $\ddagger$ M.D., M.S., F.R.C.S.
}

Brit. med. 9., 1967, 1, 18-21

In recent years experimental and clinical evidence has accumulated implying that renal erythropoietin is of importance in red-cell production under physiological and pathological conditions. It has been suggested that erythropoietin deficiency may be important in the anaemia of chronic destructive renal disease. Conversely, excessive elaboration of erythropoietin may be responsible for the polycythaemia sometimes seen in carcinoma of the kidney and other renal diseases, since high plasma levels of erythropoietin are occasionally found in the latter group of patients (Jacobson et al., 1957 ; Gurney et al., 1961 ; Penington, 1962 ; Brandt et al., 1963). Abnormally high tissue concentrations of erythropoietin have also been demonstrated in discrete pathological lesions such as tumours or cysts in the affected kidneys (Gurney et al., 1961 ; Penington, 1962). Blanshard and Smith (1964) have demonstrated increased tissue erythropoietin concentrations in distant metastases from a renal carcinoma. The role of renal erythropoietin in experimental polycythaemia is reviewed by Osnes (1959) and Penington (1962).

Of the renal lesions associated with polycythaemia the best known are carcinomata and simple cysts of the kidney (Conley et al., 1957 ; Damon et al., 1958; Jones et al., 1960). It is clear, however, that renal adenomata, polycystic kidneys, hydronephrosis, and even uterine fibroids may on occasion be accompanied by polycythaemia (Gardner and Freymann, 1958; Lawrence and Donald, 1959 ; Brandt et al., 1963 ; Smith and Riches, 1963). There has been only one previously reported association between renal artery stenosis and renal poly-

- Department of Neurology, Regional Neurological Centre, Newcastle General Hospital, Newcastle upon Tyne.

tPresent address: Department of Neurology, Massachusetts General Hospital, Boston. U.S.A.

$\ddagger$ Department of Urology, Newcastle General Hospital, Newcastle upon Tyne cythaemia in the human (Luke et al., 1965). For this reason we record the occurrence of an uncommon type of renal artery stenosis accompanied by renal hypertension and polycythaemia, which we believe to be the second case in the English literature.

\section{Case Report}

The patient, a 45-year-old man, was admitted to the Regional Neurological Centre on 13 March 1964. He gave a history of several episodes of vertigo and ataxia with staggering to the left, which had begun in 1957. He noticed that during these attacks objects appeared to move from side to side. These attacks lasted about one week and recurred at approximately yearly intervals until 1960, after which time he remained symptom-free for three years. He had two further attacks in June and November 1963, each of which lasted three weeks and was accompanied by weakness, stiffness, and sensory disturbance on the left side of the body. The latter episode was also attended by nausea and vomiting, and left him with residual weakness and stiffness in the left leg. He had no family history of essential hypertension or of occlusive vascular disease.

On examination he appeared to be in good health, but his blood: pressure was never less than 230/140 even after rest in bed. The cardiovascular system was otherwise normal. Examination of the abdomen revealed a loud blowing systolic murmur in the transpyloric plane to the left of the midline and over the left renal angle at the back. The ocular fundi showed marked vascular narrowing with arteriovenous nipping, and a single large "cotton-wool" exudate medial to the disc in the right fundus. Examination of the central nervous system showed weakness of pyramidal type with an increase in tone in the left leg. The deep tendon reflexes in the left leg were hyperactive but both plantar reflexes were flexor. There was mild cerebellar ataxia in both the upper and lower limbs on the left side. No other abnormal physical signs were found. 